Journal of Bangladesh Academy of Sciences, Vol. 34, No. 1, 23-31, 2010

\title{
ENERGY DEPENDENCE OF TOTAL CROSS SECTION FOR SCATTERING OF HELIUM ATOMS FROM ISOLATED ATOMIC STEPS
}

\author{
KAZI SHAMIM SULTANA AND M. Z. HAFIZ ${ }^{1}$ \\ Department of Physics, University of Chittagong, Chittagong-4331, Bangladesh
}

\begin{abstract}
The scattering of helium atoms from growing metal. surfaces is dominated by the interaction between incident atoms and isolated surface steps. Understanding the process has been constrained by the lack of both a realistic interaction potential and a satisfactory method for treating the scattering from a defect, which, because of the height difference of the adjoining terraces, extends to infinity. We have calculated step scattering cross sections for $\mathrm{Cu}(001)$ surface. The calculations make use of realistic potentials that reproduce the scattering from low index planes and are created from non-spherical, pairwise repulsive and attractive terms. The step scattering is isolated from that of adjoining terraces using the linearity of summation of scattering amplitudes in the single scattering regime. The results for equivalent step and terrace-site potentials give a total cross section significantly less than the experimental value. The total cross section increases with helium beam energy, contrary to the expectations that it is dominated by scattering from the attractive part of the potential. The present work confirms the prediction of our previous study that not the Van der Waals interaction but the hard wall of the scattering potential dominates the step scattering.
\end{abstract}

Key words: Energy dependence, Scattering, Helium atoms, Atomic steps

\section{INTRODUCTION}

Surface processes such as catalysis, oxidation, corrosion, thin film growth etc. are both technologically significant and scientifically interesting. The majority of modern electrical appliances would not exist without the capability of producing high quality, thin layers of insulators, semiconductors and metals. The technological need to understand complex surface processes for the manufacture of modern devices has boosted research into the understanding and measuring of phenomena at surfaces. The availability of high resolution diffractometer for helium scattering has allowed the structure of a wide range of simple and complex surfaces to be determined and the mechanism involved in surface growth to be understood. This rapid development poses both opportunities and challenges in the fundamental theoretical studies of surface structures and in the processes involved in different surface phenomena.

Currently, helium scattering has been widely used to characterize the growth of thin film (Traeger and Toennies 2004, Dastoor et al. 1992). Other recent studies (Jardine et al. 2002, Choi et al. 2000, Choi et al. 2001, Nave and Lemoine 2002) of helium scattering

\footnotetext{
${ }^{1}$ Institute of Information Technology, University of Dhaka, Dhaka-1000, Bangladesh.
} 
from adsorbate on different metal. surface were focussed on the distribution and dynamics of defects and adsorbates through observations of their total scattering cross sections on the surface. The sensitivity of helium to surface defects is large and scattering cross sections are in the range of 100-150 A (Traeger 2006) for adsorbed gases.

Step edges also exhibit very large cross sections (Verheij et al. 1985). A knowledge of the total cross sections for diffuse scattering of atoms from surface steps is extremely useful, not only to characterize randomly stepped clean surfaces, but also to study the epitaxial growth on a non-ideal substrate where the role of the surface steps becomes crucial and determines the morphology of the epitaxial layer ((Dastoor et al. 1992). The value of total cross section represents the distance normal to the step edge over which the surface is not specularly reflective for scattering of thermal helium atoms. Total step cross sections are thus defined in terms of an area per unit length and one expects that the magnitude of the total cross section of a step edge would be of the same order as the atomic dimension in the crystal face. The, measured values of the total cross section per unit length for diffuse scattering from random steps on Pt(111) for $16 \mathrm{meV}$ helium atoms (Verheij et al. 1985) and on $\mathrm{Cu}(001)$ for $63 \mathrm{meV}$ helium atoms (Sanchez and Ferrer 1987) have been found to be 12 and $13 \AA$, respectively. Therefore, the experimentally observed step edge cross section contradicts the notion that the total step cross section should be equal to the atomic dimension. (Poelsema and Comsa 1989, Verheij 1985) ascribed the significantly larger values of total cross section for diffuse scattering from steps to the dispersive forces.

It is well-known that the size of the total cross section for diffuse scattering in the gas phase is dominated by small angle deflections caused by long range dispersive forces. The analogy between diffuse scattering from adsorbed and free molecules was suggested first (Heuer and Rice 1985, Poelsema et al. (1984), Poelsema et al. (1982) by the similarity of the measured values of total cross section sizes. Accordingly, the conclusion inferred by Poelsema et al. (1982) Verheij et al. (1985) in explaining the value of total step cross section was that as in scattering from adsorbates, the cross section for diffuse scattering from steps is determined by long range dispersive forces. Therefore, the total step cross section should be bigger at low beam energy since the dispersive force which is a Van der Waals interaction becomes more important at low energies. We have carried out an investigation of the influence of the Van der Waals part of the helium-step interaction potential on scattering from an isolated-step on $\mathrm{Cu}(001)$ surface with the aid of a realistic potential model in our earlier work (Sultana 2001). The study showed very little effect of the attractive part of the potential on the step scattering and lead us to predict that total step cross section should increase with energy.

The present study is an extension of our previous work (Sultana 2001). The purpose of the study is to look at the energy dependency of the total step cross section and thereby to examine the arguments proposed by Poelsema et al. in the context of 
the large experimental value of the total step cross section. In this paper we have presented the calculated values of the total cross section for helium scattering from steps on $\mathrm{Cu}(001)$ surface at different beam energies with full helium-step interaction potential (i.e., both repulsive and attractive part) and interaction potential without the Van der Waals part.

Theoretical formalism for the calculation of total cross section

The scattering of helium atoms is determined by the interaction of the impinging atoms' charge distribution with that of the surface atoms. For low index metal. surfaces, the delocalised electrons associated with the surface metal atoms have a very low corrugation. Effectively, as far as a helium beam is concerned, the terraces are flat surfaces and exhibit little or no periodicity due to the lattice positions of the surface atoms. Therefore, the specularly reflected beam dominates the scattering from terraces. However, defects on the surface, such as atomic steps, act as diffuse scatterers; intensity is reflected away from the specular peak. Hence, as the number density of defects increases there is a corresponding increase in diffuse scattering and consequently the specular intensity falls. The loss of specular intensity with increase of defect density can be quantified in terms of a scattering cross section.

If we define a scattering cross section a associated with each $n$ such isolated defects per unit area, then the helium scattering amplitude lost is proportional to the total defect area $(\mathrm{n} \sigma)$ :

$$
\mathrm{A}=A_{0}(1-n \sigma)
$$

Therefore, the observed intensity may be written as:

$$
\mathrm{I}=I_{0}(1-n \sigma)^{2}
$$

where $A_{0}$ and $l_{0}$ are, respectively the reflected amplitude and intensity from a clean surface. A similar expression is derived for steps on the surface (Verheij et al. 1985, Poelsema and Comsa 1989). The total cross section for step scattering is given in terms of a step width $\mathrm{D}$ (i.e. $\sigma=\mathrm{D}$ ) along the step edge contour. If $\mathrm{n}$ is the total step edge length per unit area $(n=S)$, then a fraction $D S$ of the surface will scatter diffusely. Thus, in analogy with the scattering from isolated defects, such as adsorbents and monovacancies (eqn. 2), the in-phase fractional specularly reflected helium intensity is,

$$
I / I_{0}=(1-D S)^{2}
$$

where the quantity $S$ is directly related to the step density and $I_{o}$ is the specular intensity for $S \approx{ }^{`} 0$. Therefore, by experimentally determining $S$ and $I / I_{0}$ one could directly obtain $D$.

Exact information about the step density and the terrace width distribution can be obtained by analysing the specular peak profile taken under out-of-phase conditions (Verheij et al. 1985, Lent and Cohen 1984, Lu and Lagally 1982, Lapujoulade 1981, 
Poelsema et al. 1984) commonly known as a specular lattice rod scan. A schematic diagram of a typical lattice rod scan is shown in Fig. 1. The observation of an oscillatory behaviour of the specular helium peak height with varying perpendicular momentum change enables a rough estimate of the step density without additional measurements to be made. These oscillations will be visible only if the mean terrace length is not much larger than the transfer width of the instrument and not smaller than the characteristic "12 $\stackrel{0}{A}$ ". The ratio $I / I_{0}$ is obtained by measuring the in-phase specular peak height for a surface with steps and with a defect free surface. Thus by combining in- and out-of phase measurements an estimate of the cross section per unit length for diffuse scattering from a step edge can be given.

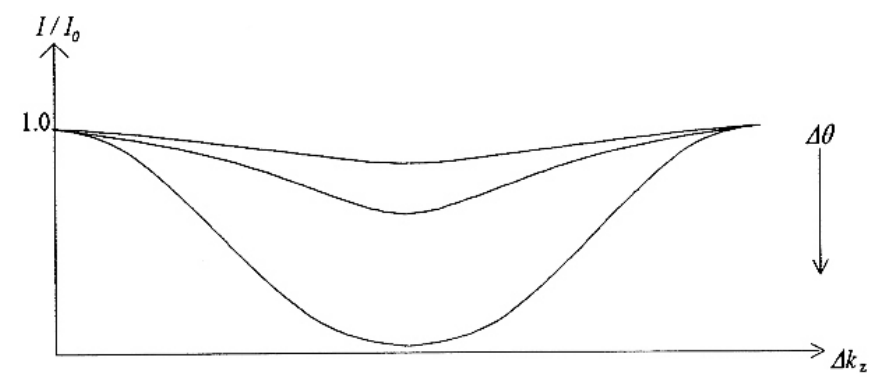

Fig. 1. A schematic diagram of a specular lattice rod scan. As the perpendicular component of the scattering vector varies, the scattering condition goes through in and out of phase conditions producing maxima and minima in the intensity curve. The difference between these two intensities decreases with increasing experimental resolution $\Delta \theta$.

By analogy with experiment it is possible to calculate the total step cross section for helium scattering from stepped surfaces with a known step density. The decay of the inphase specular intensity as the step density increases provides the value of the step density $S$ and corresponding $I / I_{0}$ value to calculate $D$. The evaluation of $D$ needs a calculation of specular intensity at the in-phase condition for surfaces with varying step density. The procedure requires the knowledge of the helium-step interaction potential and a model surface on which the step density can be easily varied.

Calculation of the total cross section for step scattering in analogy to experiment

A realistic potential for helium step scattering is needed to explore the scattering cross section in detail. One is able to evaluate a realistic helium interaction potential at a metal. surface step by summing non-spherical pair potentials using known parameters.' The method has been described elsewhere (Sultana 2001, Sultana 1997). The previously calculated potential at a $\mathrm{Cu}(001)$ step for the two cases; one with Van der Walls term i.e., the total potential (Case A) and the other without the Van der Walls term (Case B) using the same smoothing factor $\gamma=1.5$ for all of the atoms as for the atoms on a flat $\mathrm{Cu}(001)$ surface shown in Figs. 2 and 3 (Sultana 2001) have been used for the calculation of the total cross section for step scattering. 
The method of theoretically calculating the cross section for step scattering is analogous to the experimental procedure. Equation (3) has been used for the calculation. In order to calculate the cross section a two-layer regularly stepped $\mathrm{Cu}$ (001) surface as shown in Fig. 4 has been used. The surface repeats itself and the step density (the step length $\mathrm{S}$ per unit surface area) can be varied by changing the length of the repeat unit by varying the terrace length $\mathrm{L}$. The upper and lower terrace length are taken to be equal for simplicity. A series of calculations have been performed in order to apply Eqn. (3) to obtain $D$, the scattering cross section. For each in-phase scattering condition (scattering from upper and lower levels are in-phase for the beam energy used) at normal incidence of the helium beam, specular intensity $(I)$ has been calculated for different step densities. The procedure for calculating scattered intensities $(I)$ using modified sudden approximation has been described elsewhere in details (Sultana 2001, Hinch 1989). The variation of incident angle up to $30^{\circ}$ for a particular beam energy in our calculation does not give any significant change in the value of the total step cross section. Hence, only the normal incidence of the helium beam has been used in scattering calculations.

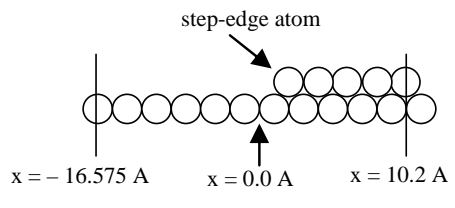

Cross-section of a $\mathrm{Cu}(100)$ up-step

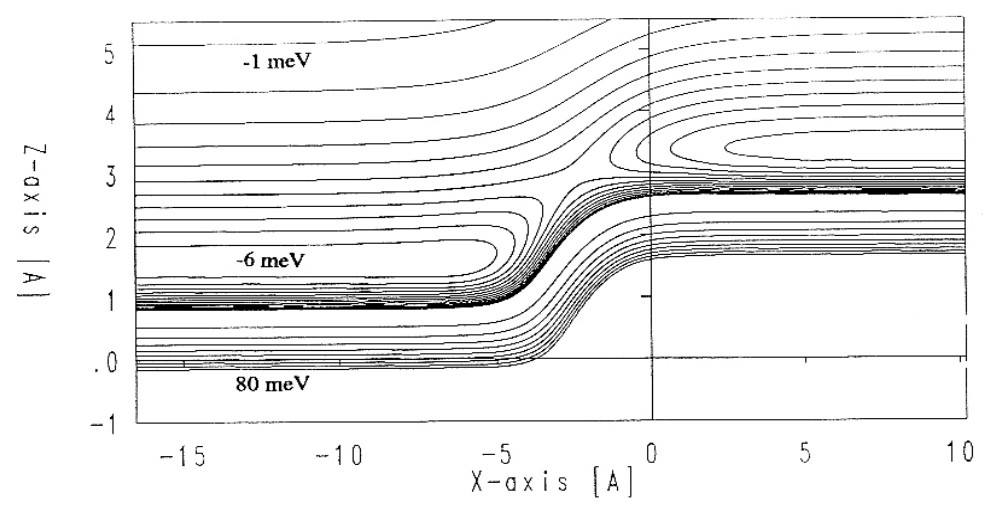

Fig. 2. Case A: Contour plot of a potential calculated using our method to represent the total helium interaction at $\mathrm{Cu}(001)$ step edge (an up step). The z-axis is perpendicular to, and the $\mathrm{x}$-axis is parallel to the surface. Step height is $1.803 \AA$ and the calculated potential minimum is $-6.4 \mathrm{meV}$. The contours -6 and $-1 \mathrm{meV}$ are drawn in steps of $0.5 \mathrm{meV}$ and between 10 and $80 \mathrm{meV}$ are drawn in steps of $10 \mathrm{meV}$. The smoothing factor used in this calculation was $\gamma=1.5$ (Sultana 2001). 


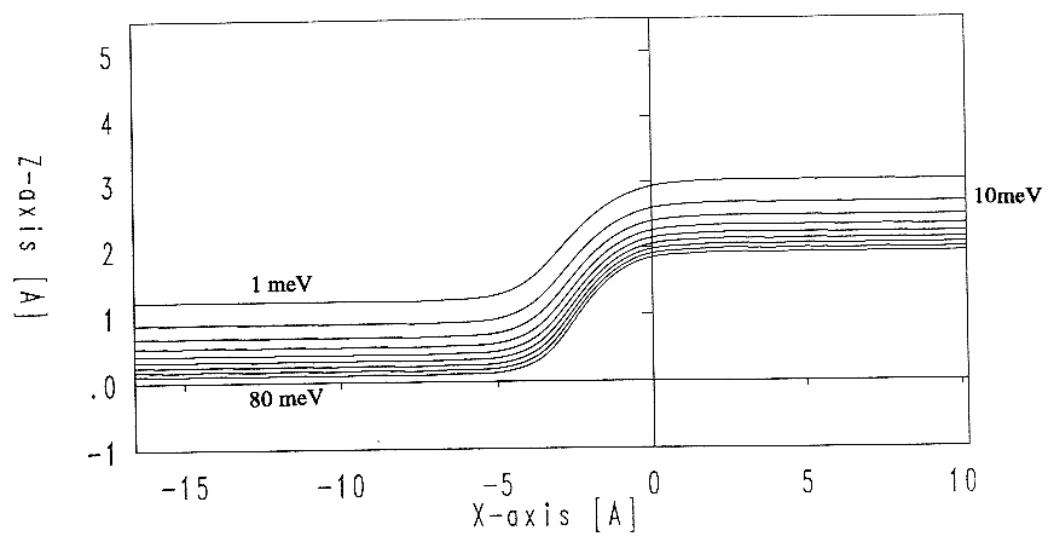

Fig. 3. Case B: This contours it also for an up step of $\mathrm{Cu}(001)$. In this case the Van der Walls term has been taked zero. The contours between 10 and $80 \mathrm{meV}$ are drawn in steps of $10 \mathrm{meV}$. Here $\gamma=1.5$ as well (Sultana 2001).

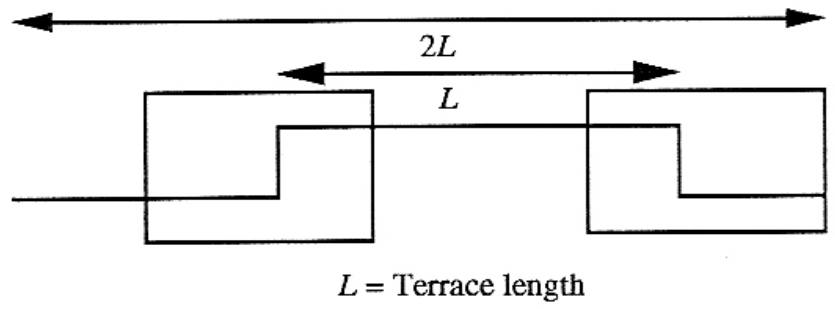

Fig. 4. A two layer regularly stepped $\mathrm{Cu}(001)$ surface model (an ideal case where the steps have sharp transitions). The step density of the surface can be varied by changing the terrace length $\mathrm{L}$. The upper terrace length is taken to be equal to the lower terrace length.

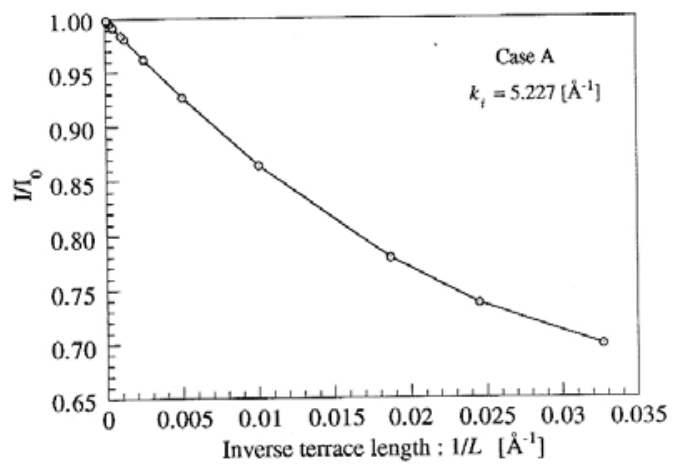

Fig. 5. Relative specular intensities calculated for the above surface for different step densities at normal incidence for a particular wave vector $\mathrm{k}_{\mathrm{c}}=5.227 / \AA$. At this energy the scattering from the upper and lower levels of the surface are at the in-phase condition. In this case the relative specular intensity $\left(I / I_{0}\right)$ decreases as expected from eqn. (3). 


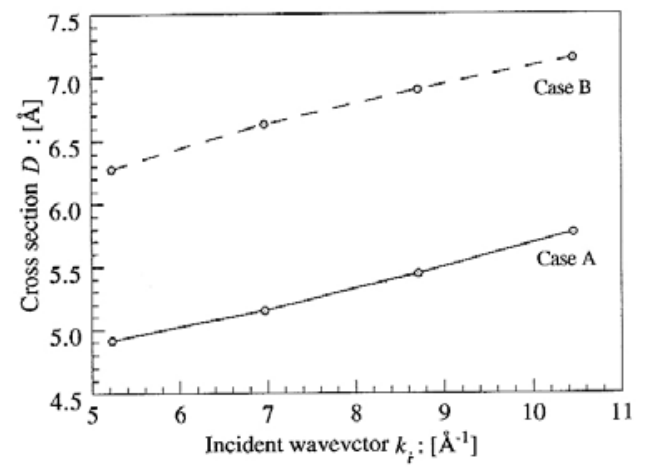

Fig. 6. Variation of the step cross section with incident wave vector ( $k i)$, equivalently, helium beam energy. It shows that the cross section for Case A is less than Case B and the cross section increases with energy.

\section{RESULTS AND DISCUSSION}

Fig. 5 shows typical results for the variation of relative specular intensity $I / I_{0}$ at a particular helium beam energy (i.e., a particular incident wavevctor ki.) with increasing step density. The scattered specular intensity (I), from a stepped surface decreases with respect to a flat surface (intensity $I_{0}$ ) due to diffuse scattering from the areas around the steps. The curve in Fig. 5 behaves as expected from expression (3) and the specular intensity decreases with step density. In real experiments, the detector used to measure the intensity has a finite width. So one does not measure intensity exactly at the specular direction but in a range of angles around the specular direction. However, the scattering close to the specular direction does not vary significantly and any effects due to finite instrumental resolution can be neglected. It has been confirmed by our calculations where we varied the instrumental resolution up to $5^{0}$ and found no significant change in the value of the total cross sections, Therefore, in this study we use calculations for an ideal experiment. The slope of the tangent drawn at zero step density $(S \approx 0)$ provides the ratio $I / I_{0}$ and corresponding step length per unit area $(S=1 / \mathrm{L})$. A cross section width $D$ can be obtained using these values in Eqn. (3). In this way the step cross section for different helium beam energies can be evaluated. Fig. 6 shows the resulting variation of cross section with incident wave vector (i.e., beam energy). The points on the curves in Fig. 6 are always calculated for specular scattering at an in-phase condition i.e., the incident wave vectors used in the calculations are chosen such that constructive interference occurs between the scattered beams from the flat terraces adjoining the step edge. The solid curve shows the total step cross section at different incident beam energies for a $\mathrm{Cu}(001)$ stepped surface using the total helium-step interaction potential (as in Fig. 2) the dashed curve is the same for helium step interaction potential without the Van der Waals term (as in Fig. 3). It can be noticed from Fig. 6 that the magnitude of the calculated cross section (around 5 to $8 \AA$ ) is significantly smaller 
than the experimental value (around $12 \AA$ ). But the most important finding is that the total cross section increases with energy in both cases contrary to the argument given by Poelsema et al. (1984). The fact that the total cross section for case A is less than case B and the energy dependence in both cases contradicts the usual notion that the Van der Waals potential is the major contributory factor to the step scattering cross section. This confirms the predictions of our previous study (Sultana 2001).

The purpose of this study was to further the present understanding of the helium scattering from growing surfaces. Our findings suggest that careful and new experiments are required to understand the origin of the larger values of total step cross section observed experimentally and also to prove the surprising but interesting finding that scattering phenomena is not dominated by the Van der waals interaction. New calculations are being carried out using the relaxation of the surface atoms and the electronic re-arrangement in the vicinity of the step. Investigation of the effect of these two factors on the total step scattering will lead to a bettering understanding of the thin film growth.

\section{REFERENCES}

Choi B. H., A. P. Graham, K. T. Tang and J. P. Toennies. 2000. Helium atom scattering from isolated Co molecules on a Pt (111) surface: experiment versus close-coupling calculations for a realistic He-Co potential. J. Chem. Phys. 112: 10538-47.

Choi B. H., K. T. Tang and J. P. Toennies. 2001. Correlation between elastic and inelastic atom scattering from single adsorbate molecules. J. Chem. Phys. 114: 2883-2886.

Dastoor P., M. Arnott, E. M. McCash and W. Allison. 1992. Initial growth morphology in molecular beam epitaxy of fcc iron on $\mathrm{Cu}\{100\}$. Surf. Sci. 272: 154.

Heuer M. and T. M. Rice, 1985. Theory of diffuse atom scattering from isolated atoms. J. Phys. BCondensed Matter. 59:299-309.

Hinch B. J. 1989. An extension of the sudden approximation for atom surface scattering with large parallel momentum transfers. Surf. Sci. 221: 346-364.

Jardine A.P. J. Ellis and W. Allison. 2002. Quasi-elastic helium-atom scattering from surfaces: experiment and interpretation. J. Phys: Condens. Matter. 14: 6173-6191.

Lapujoulade J. 1981. A statistical model for the Scattering of atoms from randomly stepped surfaces. Surf. Sci. 108: 526-548.

Lent C. S. And P. I. Cohen. 1984. Diffraction from stepped surfaces. I. Reversible surface. Surf. Sci. 139:121-154.

Lu T. M. And M. G. Lagally. 1982. Diffraction from surfaces with randomly distributed steps. Surf. Sci. 120: 47-66.

Poelsema B. and G. Comsa. 1989. In: Springer Tracts in Modern Physics. 115: 16, 17.

Poelsema B. R. L. Palmer and G. Comsa. 1984. A thermal He scattering study of Co adsorption on Pt (111). Surf. Sci. 136: 1-14.

Poelsema B. S., T. de Zwart and G. Comsa. 1982. Scattering. Cross section of low coverage Co on pt (111) for thermal He and $\mathrm{H}_{2}$ beams. Phys. Rev. Lett. 49: 578-581.

Poelsema B., L. K. Verheij and G. Comsa. 1984. Does the surface temperature influence the cross section of random isolated adsorbates in thermal He scattering? 148: 117-125. 
Sanchez A. and S. Ferrer. 1987. Cross section for diffuse scattering from random steps on Cu (100) determined by TEAS (Thermal energy atom scattering). Surf. Sci. 187: L 587-L591.

Sultana K. S. 1997. Analysis of Helium Scattering from stepped and Growing Surfaces. Ph.D thesis, Dept. of Physics, University of Combridge. Pp 34-38, 78-79.

Sultana K. S. and W. Allison. 2001. Validity of kinematic approximation in the interpretation of helium atom scattering from growing surfaces. The Chittagong Univ. J. Sci. 25(1): 51-63,

Sultana K.S. 2001. Effect of Van der Waals forces on helium scattering from isolated step edge defects. The Chittagong Univ. J. Sci. 25(1): 95-103,

Traeger F. 2006. Helium atom scattering from oxide surfaces. Chem. Phys. Chem. 7: 1006-1013.

Traeger F. and J. P. Toennies, 2004. Helium atom scattering studies of the structures and vibrations of the $\mathrm{H}_{2}$, HD and $\mathrm{D}_{2}$ monolayers on Nacl (001). J. Phys. Chem. B. 108: 14710-14725.

Verheij L. K., B. Poelsema and G. Comsa. 1985. Investigation of a randomly stepped Pt (111) surface using thermal energy atom scattering (TEAS). Surf. Sci. 162: 858-864.

(Received revised manuscript on 10 February, 2010) 